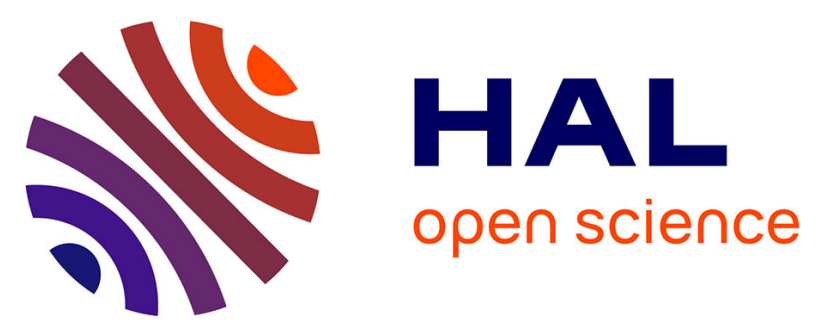

\title{
Kinetic structures of quasi-perpendicular shocks in global particle-in-cell simulations
}

Peng Bo, Stefano Markidis, Erwin Laure, Andreas Johlander, Andris Vaivads, Yuri Khotyaintsev, Pierre Henri, Giovanni Lapenta

\section{To cite this version:}

Peng Bo, Stefano Markidis, Erwin Laure, Andreas Johlander, Andris Vaivads, et al.. Kinetic structures of quasi-perpendicular shocks in global particle-in-cell simulations. Physics of Plasmas, 2015, 22, 092109 (9 p.). 10.1063/1.4930212 . insu-01255467

\section{HAL Id: insu-01255467 https://hal-insu.archives-ouvertes.fr/insu-01255467}

Submitted on 13 Jan 2016

HAL is a multi-disciplinary open access archive for the deposit and dissemination of scientific research documents, whether they are published or not. The documents may come from teaching and research institutions in France or abroad, or from public or private research centers.
L'archive ouverte pluridisciplinaire HAL, est destinée au dépôt et à la diffusion de documents scientifiques de niveau recherche, publiés ou non, émanant des établissements d'enseignement et de recherche français ou étrangers, des laboratoires publics ou privés.

\section{(ㅇ)(1) $\$$}

Distributed under a Creative Commons Attribution - NonCommercial - NoDerivatives| 4.0 


\section{AIP $\left.\right|_{\text {Physics of }}$

Kinetic structures of quasi-perpendicular shocks in global particle-in-cell simulations

Ivy Bo Peng, Stefano Markidis, Erwin Laure, Andreas Johlander, Andris Vaivads, Yuri Khotyaintsev, Pierre Henri, and Giovanni Lapenta

Citation: Physics of Plasmas 22, 092109 (2015); doi: 10.1063/1.4930212

View online: http://dx.doi.org/10.1063/1.4930212

View Table of Contents: http://scitation.aip.org/content/aip/journal/pop/22/9?ver=pdfcov

Published by the AIP Publishing

Articles you may be interested in

Quasilinear theory and particle-in-cell simulation of proton cyclotron instability

Phys. Plasmas 21, 062118 (2014); 10.1063/1.4885359

Wavenumber spectrum of whistler turbulence: Particle-in-cell simulation

Phys. Plasmas 17, 122316 (2010); 10.1063/1.3526602

Whistler turbulence: Particle-in-cell simulations

Phys. Plasmas 15, 102305 (2008); 10.1063/1.2997339

High Frequency Gyrokinetic Particle-in-Cell Simulation: Application to Heating of Magnetically Confined Plasmas

AIP Conf. Proc. 933, 475 (2007); 10.1063/1.2800535

Nonstationarity of strong collisionless quasiperpendicular shocks: Theory and full particle numerical simulations Phys. Plasmas 9, 1192 (2002); 10.1063/1.1457465

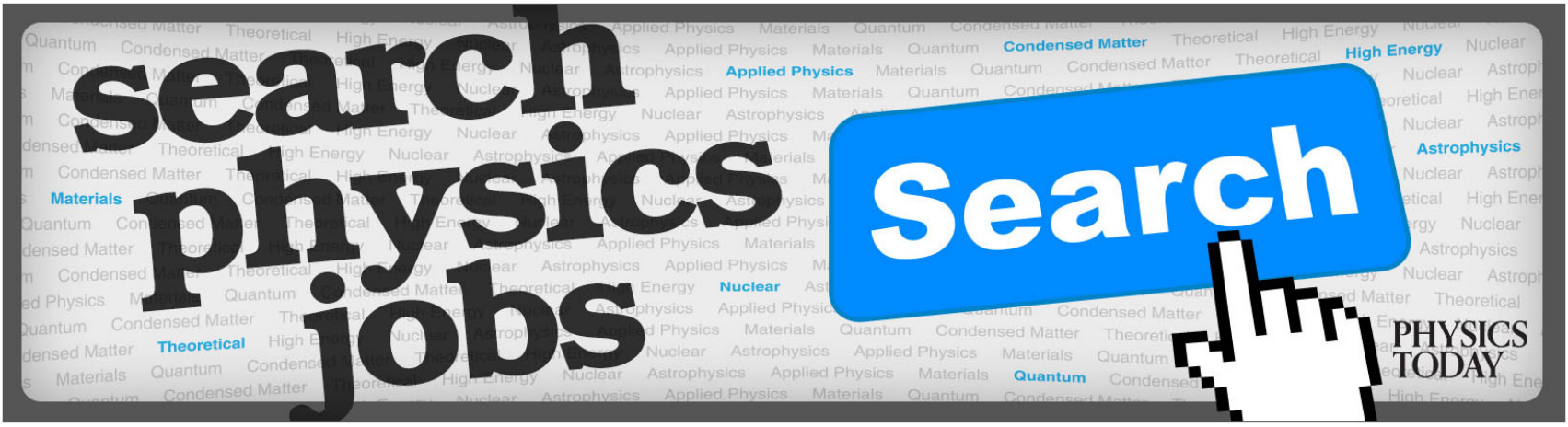




\title{
Kinetic structures of quasi-perpendicular shocks in global particle-in-cell simulations
}

\author{
Ivy Bo Peng, ${ }^{1, \text { a) }}$ Stefano Markidis, ${ }^{1}$ Erwin Laure, ${ }^{1}$ Andreas Johlander, ${ }^{2}$ Andris Vaivads, ${ }^{2}$ \\ Yuri Khotyaintsev, ${ }^{2}$ Pierre Henri, ${ }^{3}$ and Giovanni Lapenta ${ }^{4}$ \\ ${ }^{1}$ KTH Royal Institute of Technology, Stockholm, Sweden \\ ${ }^{2}$ Swedish Institute of Space Physics, Uppsala, Sweden \\ ${ }^{3}$ LPC2E-CNRS, Orléans, France \\ ${ }^{4}$ Centre for mathematical Plasma-Astrophysics, KU Leuven, Leuven, Belgium
}

(Received 10 July 2015; accepted 20 August 2015; published online 14 September 2015)

\begin{abstract}
We carried out global Particle-in-Cell simulations of the interaction between the solar wind and a magnetosphere to study the kinetic collisionless physics in super-critical quasi-perpendicular shocks. After an initial simulation transient, a collisionless bow shock forms as a result of the interaction of the solar wind and a planet magnetic dipole. The shock ramp has a thickness of approximately one ion skin depth and is followed by a trailing wave train in the shock downstream. At the downstream edge of the bow shock, whistler waves propagate along the magnetic field lines and the presence of electron cyclotron waves has been identified. A small part of the solar wind ion population is specularly reflected by the shock while a larger part is deflected and heated by the shock. Solar wind ions and electrons are heated in the perpendicular directions. Ions are accelerated in the perpendicular direction in the trailing wave train region. This work is an initial effort to study the electron and ion kinetic effects developed near the bow shock in a realistic magnetic field configuration. (C) 2015 AIP Publishing LLC. [http://dx.doi.org/10.1063/1.4930212]
\end{abstract}

\section{INTRODUCTION}

The interaction of the solar wind with a planet dipolar magnetic field leads to the formation of a magnetosphere surrounding that planet. The solar wind flow compresses the dipolar magnetic field at the sun-side and elongates it at the night-side forming a magnetotail. If the solar wind velocity is higher than the magnetosonic velocity, a bow shock forms at the day-side of the planet. The bow shock obstructs the entrance of the solar wind plasma into the magnetosphere, dissipating the energy carried by the solar wind in particle heating and acceleration. To fully understand the dissipation and particle acceleration in collisionless plasmas, a full kinetic treatment of electrons and ions is required. We carried out global Particle-in-Cell (PIC) simulations with the goal of providing a realistic magnetic field topology and consistent distribution functions to study the electron and ion kinetic structures of the bow shock.

The first Particle-in-Cell (PIC) simulations of magnetosphere date back to the $1990 \mathrm{~s}$ with the pioneering work by Buneman and Nishikawa using the Tristan code. ${ }^{1-4}$ This initial work has been extended during the last two decades by improving the performance of Tristan code and by applying it to study different kinetic aspects of magnetosphere dynamics. $^{5-9}$ These PIC simulations reproduced qualitatively all features in magnetosphere, such as magnetic reconnection and bow shock formation. However, the parameters in use are far from the realistic ones, and thus cannot provide a very accurate description of the kinetic physics in magnetospheric phenomena. In particular, unrealistic electron thermal and drift velocities were used in previous global PIC

\footnotetext{
a)bopeng@kth.se.
}

simulations. The reason for this is that Particle-in-Cell simulations with explicit discretization in time are subject to numerical constraints that require to resolve in space the Debye length. To mitigate this limitation, the electron thermal velocity is artificially increased so that Debye length results approximately equal to the grid spacing. In this work, we use an implicit PIC method that discretizes implicitly in time the Maxwell's equations and particle equations of motion. The use of an implicit PIC code allows us to choose a simulation time step and a grid spacing that are much larger than those allowed by explicit PIC codes. In particular, the numerical stability can still be retained with a grid spacing of several hundred Debye lengths. For this reason, the implicit PIC method is suited to carry out global simulations of magnetosphere, where large systems need to be simulated and the severe numerical stability constraints of the explicit PIC methods need to be avoided. In this work, we use a twodimensional geometry that allows us to resolve in space both ion and electron skin depths at a reasonable degree. For this reason, both electron and ion kinetic dynamics are accurately modeled by the implicit PIC method without the need of resolving the Debye length as in the explicit PIC approach.

Quasi-perpendicular shocks have been extensively studied with Particle-in-Cell and hybrid fluid-kinetic codes in the last decades. Excellent reviews have been presented in Refs. 10-12. In the vast majority of previous studies, quasiperpendicular shocks have been studied in simple configurations with non-curved magnetic field. In this work, we study the quasi-perpendicular region of the bow shock by investigating the kinetic structures in a non-trivial magnetic field configuration.

The paper is organized as follows. First, the global PIC simulation set-up and parameters in use are introduced. 
The magnetosphere formation and the bow shock kinetic structures are presented in Section II. The results of the global PIC simulations and analysis of the bow shock structures are presented in Section III. Section IV discusses the main findings and concludes the paper.

\section{SIMULATION PARAMETERS}

Global Particle-in-Cell simulations are carried out in a reduced $2 \mathrm{D} 3 \mathrm{~V}$ configuration, where there are two coordinates for space and three coordinates for velocity, current, and electromagnetic field. The Geocentric Space Magnetospheric (GSM) coordinate system is in use. The $x$ direction is along Sun-planet direction and the $z$ direction is along magnetic dipole moment in the South-North direction. The $y$ direction is the out-of-plane direction and is the ignorable coordinate in these simulations. The simulation box is $L_{x} \times L_{z}=120 d_{i} \times 240 d_{i}$. The ion inertial length is $d_{i}=c / \omega_{p i}$, with $c$ the speed of light in vacuum, the ion plasma frequency $\omega_{p i}=\sqrt{4 \pi n_{0} e^{2} / m_{i}}, e$ the elementary charge, and $m_{i}$ the ion mass. A reduced ion-electron mass ratio $m_{i} / m_{e}=64$ is used. The grid consists of $1024 \times 2048$ cells. The grid spacing is $\Delta x=\Delta z=0.1172 d_{i}=0.9375 d_{e}$.

The Interplanetary Magnetic Field (IMF) intensity is $B_{I M F}=0.005 m_{i} \omega_{p i} / e$ and it is only in the Northward $z$ direction. The angle $\Theta_{B n}$ between the upstream $\mathbf{B}_{I M F}$ and the shock normal changes depending on the location of the curved bow shock front. For this reason, a bow shock consists of quasi-perpendicular and quasi-parallel regions, for which $\Theta_{B n}>\pi / 4$ and $\Theta_{B n}<\pi / 4$, respectively. In this work, we focus on the quasi-perpendicular region, where $\Theta_{B n}$ is close to $\pi / 2$.

The solar wind velocity is $v_{0}=0.02 c$. The electron thermal velocity is $0.028 c$. Electrons and ions have the same temperature. In this configuration, the ratio between kinetic and magnetic pressure is $\beta=0.98$. The solar wind magnetosonic Mach number is $M=2.84$. The first critical Mach number categorizes the shock into sub- and super-critical shock and it depends on the plasma $\beta$, angle $\Theta_{B n}$, and the ion to electron mass ratio. ${ }^{12,13}$ Taking into account the simulation parameters, the shock is super-critical for the quasi-perpendicular region with $\Theta_{B n} \approx \pi / 2{ }^{13}$ We note that $M=2.84$ is also higher than whistler critical number. ${ }^{14}$ The simulation time step is $\Delta t=0.1 \omega_{p i}^{-1}=0.8 \omega_{p e}^{-1}$, where $\omega_{p}$ is the plasma frequency. The total simulation time is twice the solar wind transit time in the simulation box. This corresponds to 120000 computational cycles. At this time, the simulated magnetosphere reaches a quasi-steady state when the main magnetosphere features, such as magnetopause stand-off distance, do not change in time. Together with a uniform IMF magnetic field, a convective electric field $\mathbf{E}=-\mathbf{v}_{0} \times \mathbf{B}_{I M F}$ is initially imposed in the simulation box. In simulations of magnetospheres, the magnetic field is the combination of a varying magnetic field $\mathbf{B}_{\text {int }}$ and a fixed dipolar magnetic field $\mathbf{B}_{\text {ext }}:{ }^{15}$

$$
\mathbf{B}=\mathbf{B}_{\text {int }}+\mathbf{B}_{\text {ext }} .
$$

The 2D fixed dipolar magnetic field, aligned with the $z$ axis and centered at the $\left(x_{0}, z_{0}=60 d_{i}, 120 d_{i}\right)$ point, is

$$
\begin{gathered}
B_{e x t, x}=D \frac{2\left(x-x_{0}\right)\left(z-z_{0}\right)}{r^{4}} \\
B_{e x t, z}=D \frac{2\left(z-z_{0}\right)^{2}-\left(x-x_{0}\right)^{2}}{r^{4}},
\end{gathered}
$$

where $r=\sqrt{\left(x-x_{0}\right)^{2}+\left(z-z_{0}\right)^{2}}$ and $D=0.2 m_{i} \omega_{p i} / e$ is the dipole strength. In the $2 \mathrm{D}$ dipole case, the magnetic field decreases as $r^{-2}$ instead of $r^{-3}$ (3D case). ${ }^{16}$

Inflow boundary conditions are imposed at $x=0$, while open outflow boundary conditions are imposed at all the other directions. The implementation details of the inflow and outflow boundary conditions are reported in Ref. 17. Absorbing boundary conditions are imposed on the planet surface: particles are removed when their position is in the planet region. A uniform zero net charge density is imposed in the planet region.

A relativistic particle mover with an adaptive subcycling is in use to update the particle position and velocity accurately and efficiently. ${ }^{17,18}$ Simulations are carried out with the massively parallel implicit PIC $i P I C 3 D$ code, ${ }^{19}$ running on 2048 cores of the Beskow Cray XC40 supercomputer at KTH for $6 \mathrm{~h}$.

\section{RESULTS}

In this section, we first present the overall structure of the bow shock formation in the global PIC simulations. Then, we focus on identifying the wave activity in the quasiperpendicular bow shock region. Finally, we analyze the distribution functions and phase space for ions and electrons close to the quasi-perpendicular shock regions with the goal of determining particle heating and acceleration.

\section{A. Overall simulation evolution}

The simulation box is initialized with a uniform drifting plasma impinging a planet with a dipolar magnetic field. The interaction of the solar wind with a planet magnetic field rapidly creates a magnetosphere. Figure 1 shows different stages of the magnetosphere formation in our PIC simulation: each panel of Figure 1 presents a contour plot of the electron density with superimposed magnetic field lines near the bow shock region at different simulation time steps. The evolution of the whole system is visible. The initial uniform density is clear from the first panel. As the simulation continues, a magnetosphere gradually forms over a $10000 \omega_{p i}^{-1}$ period. After this time, minimal changes in the magnetosphere features, such as the stand-off magnetopause distance, are observed, indicating the simulation reached a quasi-steady state. The on-line supporting multimedia material presents the evolution of the electron density and magnetic field lines. We note that the magnetosheath in this two-dimensional simulation is thicker than the magnetosheath in more realistic three-dimensional simulations. ${ }^{17}$

To determine the location and thickness of the shock in our simulation, we profile the magnetosonic Mach number, magnetic field, electron and ion densities at time $t=12000 \mathrm{\omega}_{p i}^{-1}$ along the line $z=L_{z} / 2$ and $x=40-50 d_{i}$ in Figure 2. In fact, a rapid change of these quantities can reveal the presence of 

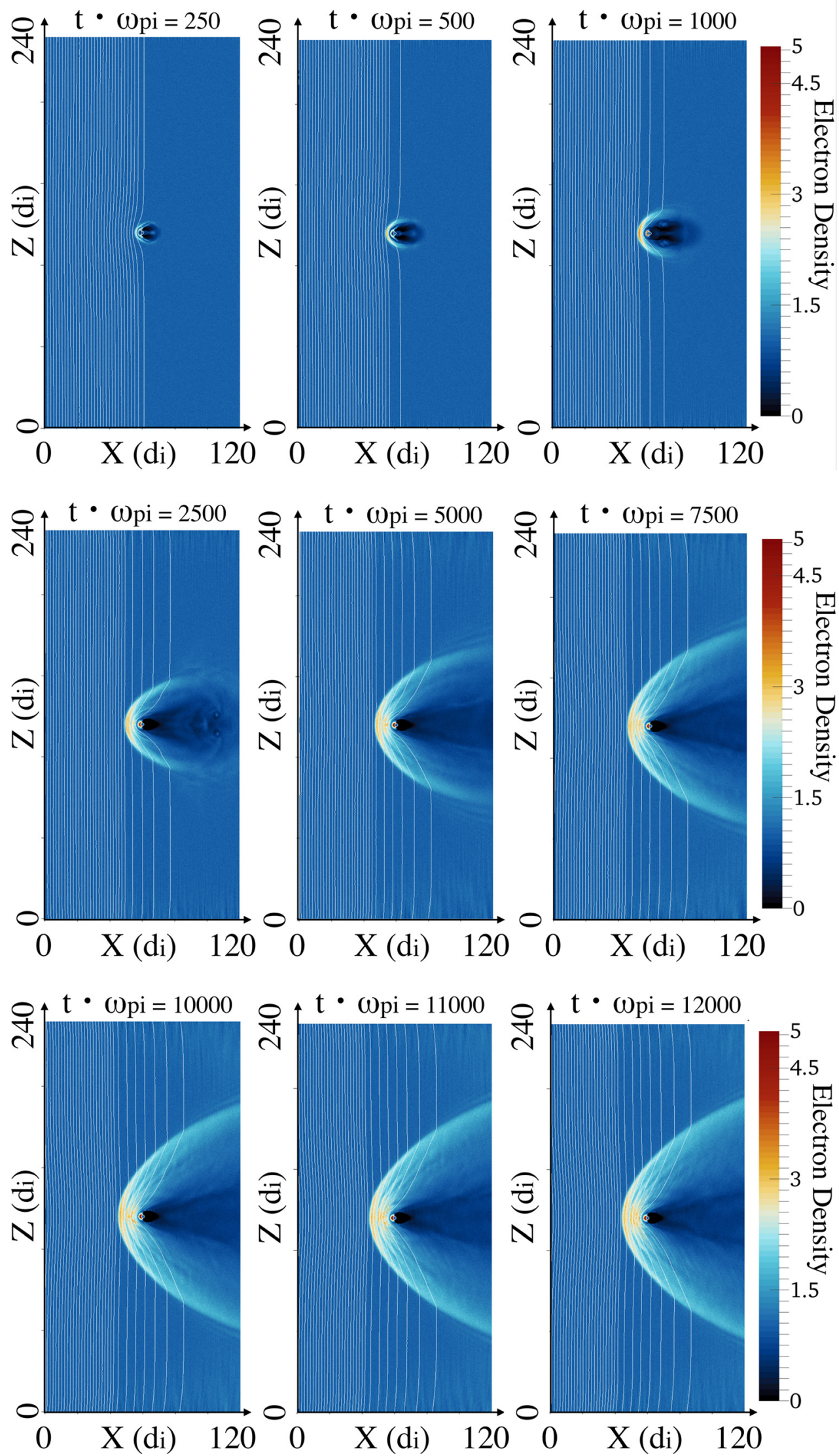

FIG. 1. Contour plots of the electron density with superimposed magnetic field lines at different times. A magnetosphere with a bow shock rapidly forms. After $10000 \omega_{p i}^{-1}$, the simulation reaches a quasi-steady state. (Multimedia view) [URL: http://dx.doi.org/10.1063/1.4930212.1] 


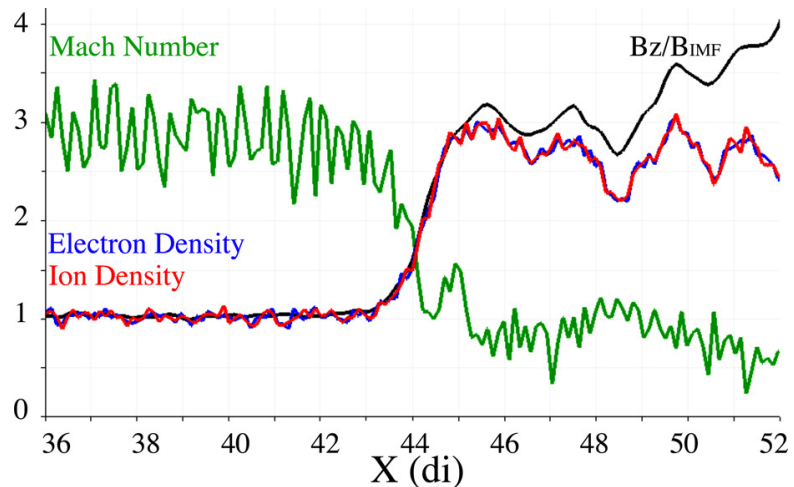

FIG. 2. The magnetosonic Mach number, $z$ component of magnetic field (normalized over the IMF intensity), electron and ion densities at time $t$ $=12000 \omega_{p i}^{-1}$ are plotted for $x=36-52 d_{i}$ and $z=L_{z} / 2$ in green, black, blue, and red colours, respectively. Electron and ion densities overlap mostly. Particle density and $z$ component of magnetic field increase by approximately three times, while Mach number decreases rapidly around $x=43.5 d_{i}$. Mach number drops below one around $x=45 d_{i}$.

bow shock. The solar wind flow from super-magnetosonic (magnetosonic Mach number $=2.8$ ) to sub-sonic in proximity to the bow shock. The $z$ component of the magnetic field and the particle densities both increase by a factor of 3 after the bow shock. The overlapping lines for electron and ion densities in Figure 2 indicate no occurrence of net charge. After the shock, the magnetic field shows oscillations in $z$ component and the presence of a trailing wave train. Figure 2 allows us to approximately determine the scale length of the bow shock in our simulation to be $1 d_{i}$ and the wavelength of the trailing wave to be $2 d_{i}$.

The structure of the electromagnetic field near the bow shock $\left(x=36-52 d_{i}\right.$ and $\left.z=90-150 d_{i}\right)$ is investigated. Figure 3 shows a contour-plot of the three components of the magnetic field in this region. The strongest component is in the $z$ direction as both IMF and dipolar magnetic field moments are along the $z$ direction. The out-of-plane ( $y$ direction) magnetic field component shows the presence of the trailing wave train.

Figure 4 shows the contour plot of the three components of electric field. The strongest component is in the $x$ direction and is capable of accelerating ballistically plasma particles.

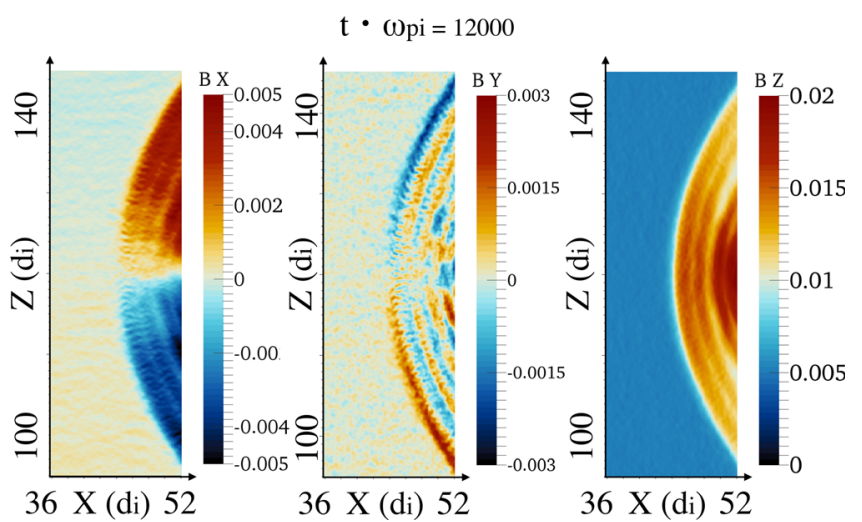

FIG. 3. Contour plot of the three magnetic field components near the bow shock at time $t=12000 \omega_{p i}^{-1}$. The unit of the magnetic field is $m_{i} \omega_{p i} / e$. A clear signature of the trailing wave train is visible in the out-of-plane $B_{y}$ component of the magnetic field.

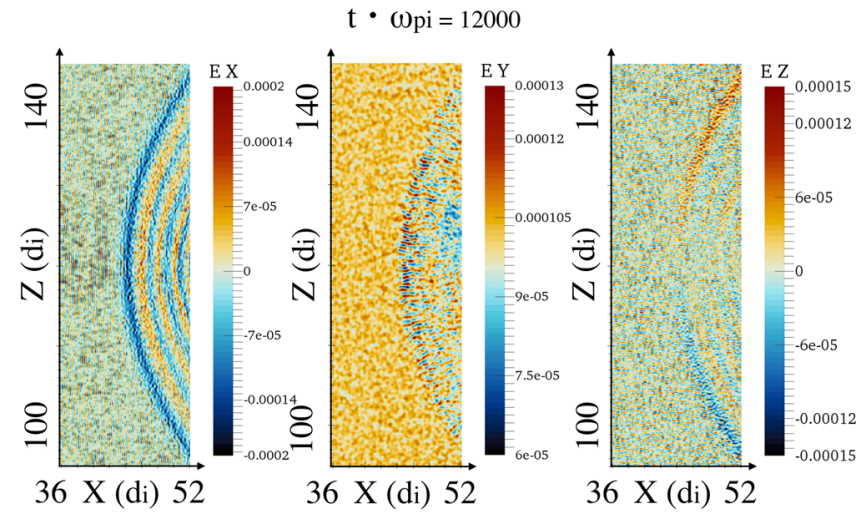

FIG. 4. Contour plots of the three electric field components near the bow shock at time $t=12000 \omega_{p i}^{-1}$. A short wavelength wave along the magnetic field lines is visible in $E_{y}$ component.

An analysis of the $x$ component confirms the presence of the trailing wave train. A wave propagating along the magnetic field line is visible in the $y$ component with wavelength approximately $2-3 d_{e}$.

A spectral analysis has been carried out to identify the nature of the wave propagating along the magnetic field line at the edge of the bow shock (visible in the second panel of Figure 4). A numerical dispersion relation has been calculated by taking a two-dimensional Fast Fourier Transform (FFT) of $E_{y}$ values sampled every $0.2 \omega_{p i}^{-1}$ along the magnetic field line. Figure 5 shows a contour plot of the absolute value of the two dimensional FFT results. The angular wave number $k$ is on the $x$ axis and expressed in $1 / d_{i}$ units. The angular frequency $\omega$ is on the $y$ axis and is expressed in terms of the local electron cyclotron frequency $\left(\Omega_{c e}\right)$, obtained from the local magnetic field. Two black lines are superimposed to the contour plot in Figure 5. The first black dashed line represents the parallel whistler dispersion relation

$$
\omega^{2}=c^{2} k^{2}-\frac{\omega_{p}^{2}}{1-\Omega_{c e} / \omega} .
$$

The second black dotted line represents the electron cyclotron frequency that is the cut-off frequency for whistler

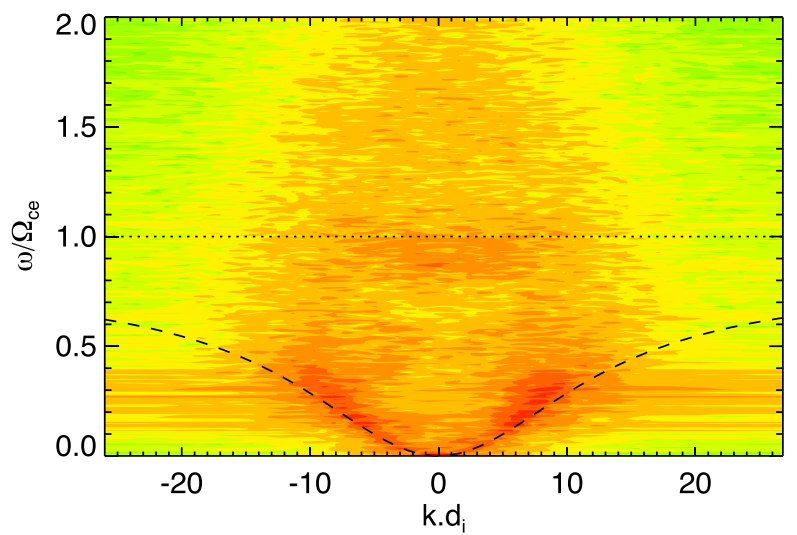

FIG. 5. Numerical dispersion relation in the bow shock region obtained from a 2D FFT of $E_{y}$. The black dashed line represents the analytical parallel whistler dispersion relation, while the black dotted line represents the electron cyclotron frequency that is the cut-off frequency for whistler waves. 


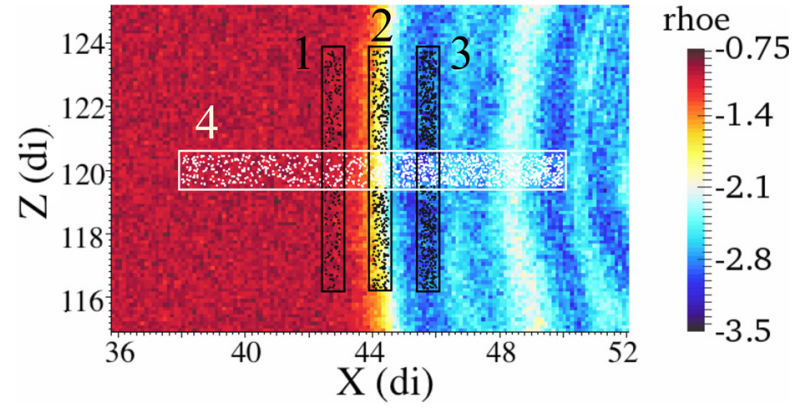

FIG. 6. Distribution functions are studied in region 1(upstream), 2 (shock ramp), and 3 (downstream in the trailing wave train area) in black dot boxes. Particle phase space is studied in region 4, the white dotted box. The electron charge density at time $t=12000 \omega_{p i}^{-1}$ is plotted in the background to ease the identification of the different regions.

waves. This cut-off is due to the wave resonance with the gyrating electrons. By comparing the numerical and theoretical dispersion relation of parallel whistler waves, it is possible to identify the waves propagating along the magnetic field lines at the edge of the bow shock as whistler waves. Together with the presence of whistler waves, we also detect electron cyclotron waves visible as a red patch localized at $\omega=\Omega_{c e}$ in the numerical dispersion relation of Figure 5 .

\section{B. Ion kinetic physics}

The use of a PIC code allows us to study the particle distribution functions and phase space. These quantities can be reconstructed from particle position and velocity and can provide important information to determine plasma acceleration and heating. Distribution functions and phase space are studied in four regions illustrated in Figure 6. Region 1 is at the upstream the shock, region 2 is at the shock ramp, and region 3 is located in the downstream region where the trailing wave train is present. Region 4 encompasses all the bow shock area.

We first study the ion kinetic physics near the bow shock. The three different panels in Figure 7 present the ion distribution function in the perpendicular directions $\left(f\left(v_{x}\right)\right.$ and $\left.f\left(v_{y}\right)\right)$ and in the parallel direction $f\left(v_{z}\right)$ ). Each panel shows the distribution functions in three regions: region 1 (upstream) in red color, region 2 (shock ramp) in green color, and region 3 (downstream) in blue color.

An analysis of the ion $f\left(v_{x}\right)$ distribution functions (first panel of Figure 7) indicates that solar wind ions are decelerated in the $x$ direction from $v_{x}=0.02 c$ to $v_{x} \approx 0.002 c$ when moving from the upstream to the downstream. This is clear from the shift of the $v_{x}$ peaks in the upstream and downstream distribution functions. The downstream $f\left(v_{x}\right)$ has a larger width (standard deviation) than the upstream $f\left(v_{x}\right)$, revealing that ions are heated. The downstream ion $f\left(v_{x}\right)$ distribution function presents a long tail, reaching $v_{x} \approx 0.04 c$ that is twice the initial ion solar wind velocity. The ion $f\left(v_{x}\right)$ distribution function in shock ramp region presents two peaks: one peak corresponds to the decelerating solar wind population at $v_{x} \approx 0.015 c$; the second peak corresponds to a counter-streaming ion populations moving at a bulk velocity $v_{x} \approx-0.005 c$ as a result of specular reflection of ions at downstream.

A comparison of the upstream and downstream ion $f\left(v_{y}\right)$ distribution functions (red and blue distribution functions in the second panel of Figure 7) shows that the initial Maxwellian distribution in $y$ direction flattens and widens, revealing that the ions are strongly accelerated in the perpendicular $y$ direction. In the shock ramp region, the ion $f\left(v_{y}\right)$ distribution function shows a long flat-top tail, reaching $v_{y} \approx 0.035 c$. This long tail corresponds to ion acceleration in the perpendicular $y$ direction. Ions are accelerated in the $y$ direction by Shock Drift Acceleration at the shock front with some ions reflected at the shock and moving upward. ${ }^{20,21}$

The last panel of Figure 7 shows the ion $f\left(v_{z}\right)$ distribution functions. In this case, no relevant changes are observed in the distribution functions, which are all approximately Maxwellian in the three regions. This fact indicates that there is no ion parallel heating or acceleration mechanism in place in the proximity of the bow shock. In three-dimensional PIC simulations, additional heating and acceleration mechanisms might be present.

The ion phase space in region 4 has been studied to determine possible ion heating and acceleration. The three panels of Figure 8 show the ion phase space with particle $x$ coordinate on the $x$ axis and $v_{x}$ (top panel), $v_{y}$ (middle panel), and $v_{z}$ (bottom panel) on the $y$ axis, respectively. A total of 103726 ion positions and velocities in region 4 are used to reconstruct the ion phase space. The phase space has been computed by discretizing the phase in $x-v$ bins and sum up particle counts in each bin. Figure 8 shows a contour-plot of the discretized $x-v$ space. Each color pixel represents the particle count in the bin.

An analysis of the $x-v_{x}$ phase space (top panel of Figure 8) reveals that a small part of the ion population is
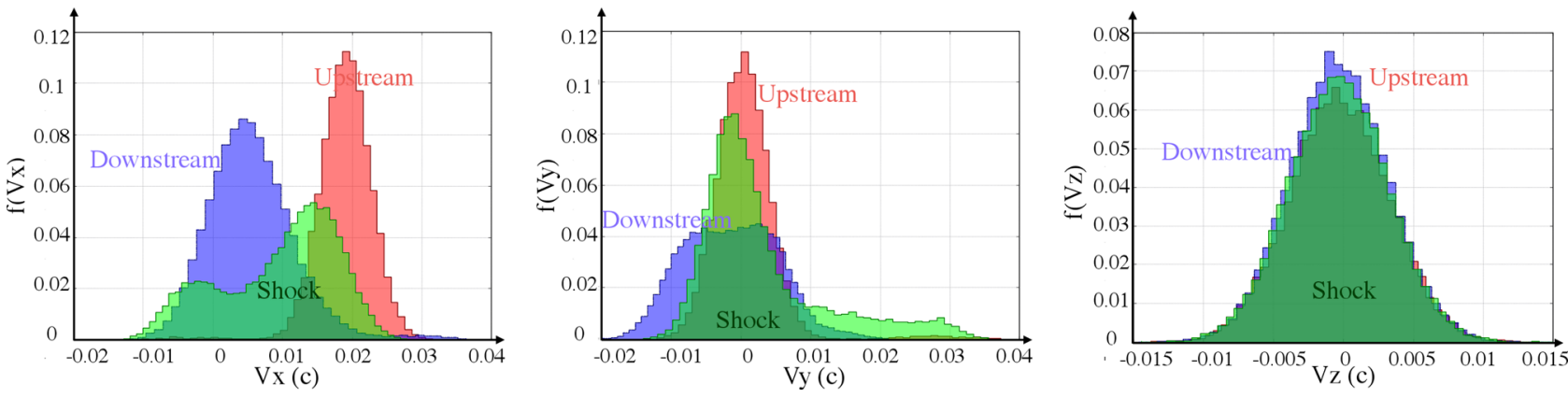

FIG. 7. The ion $f\left(v_{x}\right), f\left(v_{y}\right)$, and $f\left(v_{z}\right)$ distribution functions at time $t=12000 \omega_{p i}^{-1}$ are presented in the left, middle, and right panels, respectively. Each panel shows the ion distribution function in regions 1 (red color), 2 (green color), and 3 (blue color). 


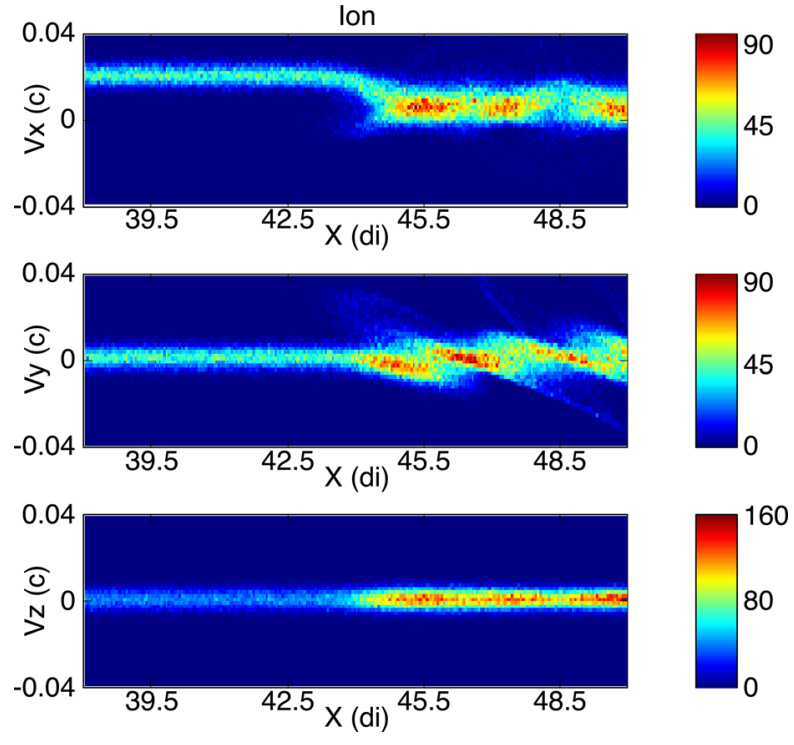

FIG. 8. Contour-plot of the ion count in region 4 at time $t=12000 \omega_{p i}^{-1}$ to represent $x-v_{x}$ (top panel), $x-v_{y}$ (middle panel), and $x-v_{z}$ (bottom panel) ion phase spaces, respectively. The top panel shows the ion specular reflection at the bow shock. Strong ion acceleration occurs in the out-ofplane $y$ direction (middle panel).

reflected at the bow shock and propagates in the negative $x$ direction for a distance of approximately $1 \mathrm{di}$. The ion plasma population crossing the bow shock is heated in the $x$ direction (velocity spread in the $v_{x}$ direction) and accelerated/decelerated at approximately $x=47.5 \mathrm{di}$ and $x=48.5 \mathrm{di}$. These acceleration/deceleration are likely due to electric field $x$ component. A study of the $x-v_{y}$ phase space (middle panel of Figure 8) shows strong acceleration in the out-of-plane $y$ direction at $v_{y}= \pm 0.04 \mathrm{c}$. We note that this out-of-plane acceleration occurs in correspondence of the trailing wave train at the shock downstream. The $x-v_{z}$ phase space does not show any sign of acceleration or heating in the parallel direction while it shows a rapid increase of the particle density after the bow shock consistently with the results of Figure 2. In summary, we observe only ion acceleration and heating only in the perpendicular directions.

\section{Electron kinetic physics}

Different from hybrid codes, the use of full PIC codes allows us to study the electron acceleration and heating at the bow shock. The electron distribution functions in the
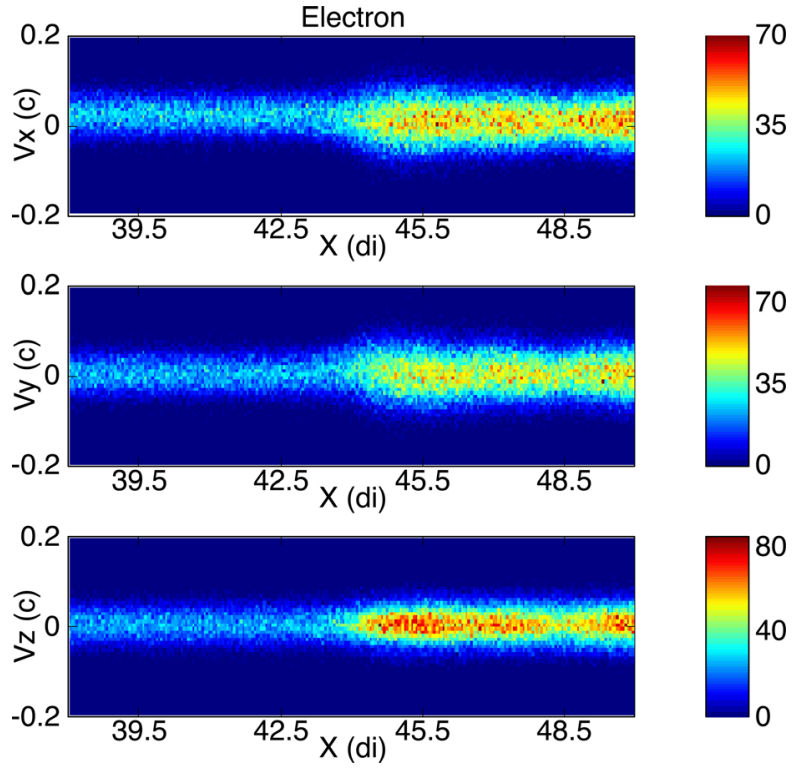

FIG. 10. Contour-plot of the electron count in region 4 at time $t=$ $12000 \omega_{p i}^{-1}$ to represent the $x-v_{x}$ (top panel), $x-v_{y}$ (middle panel), and $x-v_{z}$ (bottom panel) electron phase spaces, respectively. Electron heating is visible in the perpendicular directions (top and middle panels).

three directions have been studied in regions 1 (upstream) and 3 (downstream) and presented in Figure 9.

The left panel of Figure 9 shows that the bulk electron velocity in the $x$ direction reduced from $0.02 c$ in the upstream to approximately $0.005 c$ in the downstream. The electron heating in the $x$ direction is clear from the increased width of $f\left(v_{x}\right)$ in the downstream. Different from the ion distribution, we do not observe long tails in the perpendicular distribution functions. For this reason, it is likely that the mechanisms for strong electron acceleration are not present in our simulations. The electron $f\left(v_{z}\right)$ in regions 1 and 3 is presented in the right panel of Figure 9. The lack of considerable changes in the electron $f\left(v_{z}\right)$ shows that there is no electron heating and acceleration in the parallel direction.

The electron phase space in region 4 has been reconstructed following the same procedure to calculate the ion phase space. A total of 103617 electrons are used to reconstruct the phase space. The top and middle panels of Figure 10 present the electron phase space in the perpendicular directions, $x-v_{x}$ and $x-v_{y}$. The perpendicular electron heating is visible from the increased width of the phase space
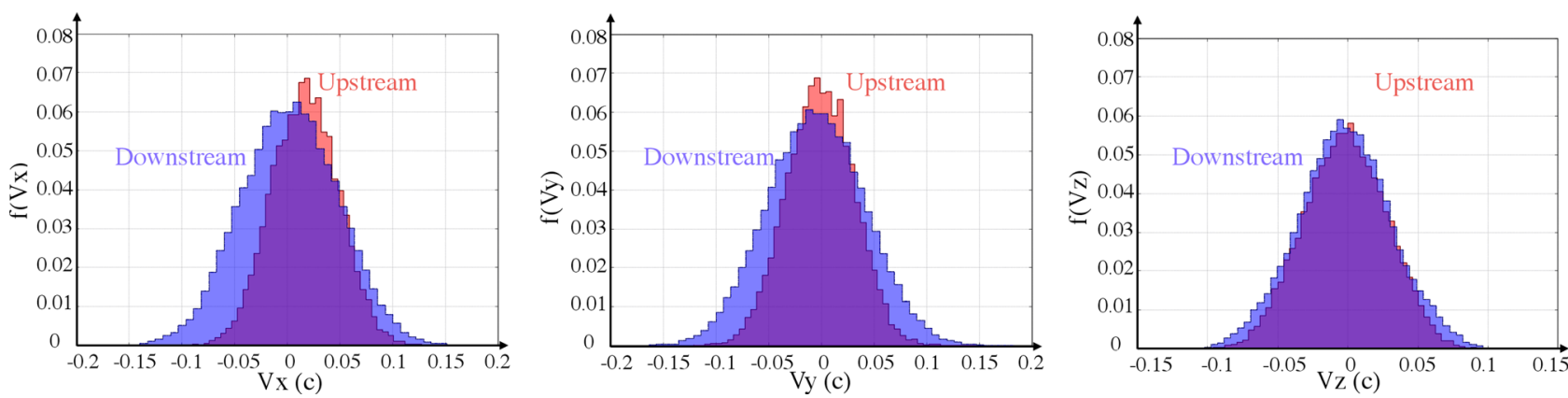

FIG. 9. The electron $f\left(v_{x}\right), f\left(v_{y}\right)$, and $f\left(v_{z}\right)$ distribution functions at time $t=12000 \omega_{p i}^{-1}$ are presented in the left, middle, and right panels, respectively. Each panel shows the ion distribution function in upstream region 1(red color) and downstream region 3 (blue color). 

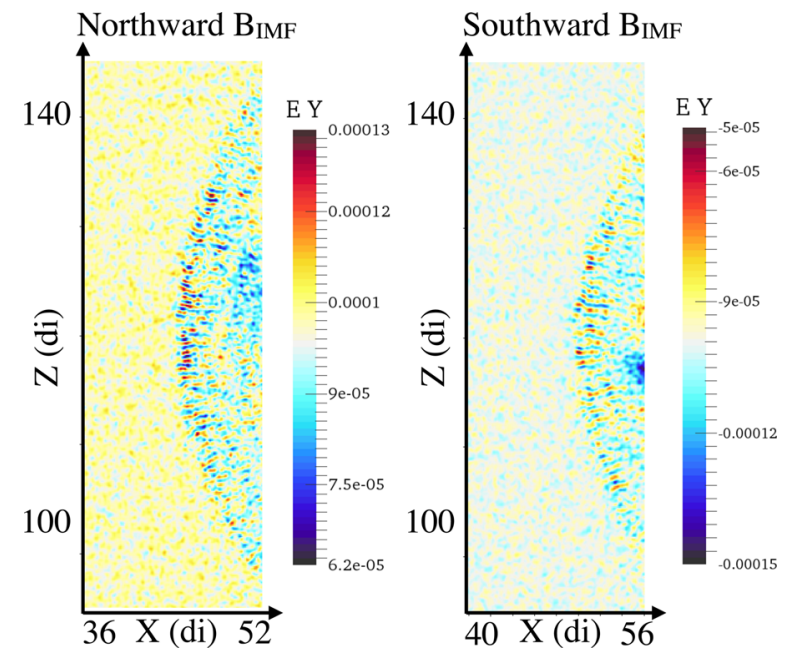

FIG. 11. Ey contour plot in the bow shock region for the Northward (left panel) and Southward (right panel) IMF PIC simulations. The whistler waves (short wavelength wave propagating along the magnetic field lines) are present in both simulations.

election beam in these two panels. The bottom panel shows the electron $x-v_{z}$ phase space in region 4 . It is clear that electron density increases during the crossing of the bow shock.

In addition, we studied the electron distribution functions and phase spaces along the magnetic field lines to determine if electrons interact with whistler waves. However, we did not find any evidence of electron scattering by whistler waves.

\section{DISCUSSION AND CONCLUSIONS}

We simulated the global interaction of the solar wind with a magnetosphere with a 2D3V PIC code and studied the kinetic collisionless physics in super-critical quasi-perpendicular shocks. The global simulation provided a realistic magnetic field topology and a self-consistent distribution function with electromagnetic field. After an initial simulation transient, a collisionless bow shock forms as a result of the interaction of super-magnetosonic solar wind with a planetary magnetic dipole. The focus of this work is to study the kinetic structures of the quasi-perpendicular shock region. Future work will extend to investigate the quasiparallel shocks. In our simulation, the shock is super-critical in the quasi-perpendicular shock region. Specularly reflected ions are found in the simulations as a sign of supercritical shock. This is consistent with the fact that super-critical shocks cannot be maintained by dissipation alone but require a decrease of ions crossing the shock ramp. This is achieved by reflecting particles in the upstream.

The thickness of the bow shock is approximately $1 d_{i}$. This result is in agreement with observations of the quasiperpendicular bow shock thickness from Cluster spacecrafts. ${ }^{22,23}$ A trailing wave train is observed after the shock ramp. The trailing wave train at downstream with magnetic field overshoots followed by undershoots is a characteristic of super-critical quasi-perpendicular shocks. ${ }^{24-26}$ We note that the trailing wave is not damped. We believe that this might be an effect of imposed boundary conditions on the planet surface and/or of the two dimensional geometry of the simulation.

The out-of-plane component of the electric field shows a short wavelength wave propagating along the magnetic field lines at the downstream edge of the shock ramp. Because these waves propagate along the magnetic field and not across it, it is unlikely that these waves are lower-hybrid waves. ${ }^{27}$ We identified these waves as whistler waves by performing spectral analysis. We have carried out an additional simulation of supercritical quasi-perpendicular bow shock with Southward IMF to determine if the presence of whistler waves depends on the direction of the IMF. Figure 11 shows the $E_{y}$ contour plot in the bow shock region for the Northward (left panel) and Southward (right panel) IMF PIC simulations. In the case of Southward IMF, magnetic reconnection occurs at the dayside magnetopause. ${ }^{28,29}$ Note that the right panel of Figure 11 does not include the region of space where magnetic reconnection occurs. Therefore, magnetic reconnection is not visible in the plot. By comparing the two panels, it is clear that the whistler waves are present in both simulations. The origin of the whistler waves at the bow shock downstream still needs to be investigated and identified. Possible mechanisms to generate whistler waves are electron temperature anisotropy and beams. ${ }^{30}$ While we found electron temperature anisotropy in parallel and perpendicular directions, electron beams were not present at the downstream of the shock. We note that whistler wave can be generated by "interface instability" caused by ion dynamics. ${ }^{31}$ Future work will investigate the cause of the whistler waves at the bow shock downstream.

By studying the phase space in a parallel direction near the bow shock, we have not found any evidence of particle heating due to the interaction of whistler waves and particles. In addition to the whistler waves, we found the presence of electron cyclotron waves in the same region. Ions and electrons are likely heated and accelerated in the perpendicular directions by the SDA. ${ }^{20,21,32}$

Ions crossing the shocks are strongly accelerated in the perpendicular out-of plane $y$ direction in the shock downstream region. This acceleration can be associated with the presence of the bow shock electromagnetic structures with strong spatial gradients of the electric field. ${ }^{33,34}$ This configuration can lead to ion gyro-phase breaking and acceleration if the following condition is satisfied:

$$
\frac{1}{\Omega_{c} B_{z}}\left|\frac{\partial E_{x}}{\partial x}\right| \geqslant 1 .
$$

Since $\Omega_{c}$ decreases linearly with charge over mass ratio of the plasma species, ions are more likely to be accelerated than electrons. Using the simulation parameters and results, $\frac{1}{\Omega_{c} B_{z}}\left|\frac{\partial E_{x}}{\partial x}\right| \approx 1 \gtrsim 1$ and therefore ions can be accelerated by this mechanism.

One limitation of this work is the use of a two dimensional geometry. A reduced geometry allows us to resolve in space both ion and electron scales at a reasonable degree. However, this reduced dimensionality does not allow plasma instability to develop on the equatorial plane, limiting the exchange of mass, momentum and energy between the solar 
wind and magnetosphere. For instance, in three-dimensional simulations, solar wind can enter at magnetopause flanks through Kelvin-Helmholtz instability. ${ }^{35}$ In addition, more realistic boundary conditions should be applied on the surface of the planet. Due to these simulation approximations, the magneto-sheath thickness (Figure 1) results larger than the one reported by other simulations and observations.

Despite the use of the implicit PIC method allowed us to use relatively small electron thermal velocities, the electron thermal velocities in our simulations are higher than typical solar wind electron velocity. The solar wind electron thermal velocity at $1 \mathrm{AU}$ is $v_{\text {the }} \approx 0.005 c$ (Ref. 36), while we used $v_{\text {the }}=0.028 \mathrm{c}$. We retained realistic plasma $\beta$ and solar wind Mach number. Another relevant approximation that affects the bow shock structure is the use of a reduced ion-electron mass ratio. Reference 12 discusses the effects of using reduced mass ratio in PIC modeling quasi-perpendicular shocks. This work is an initial effort to study the electron and ion kinetic effects developed in proximity to the bow shock in a realistic magnetic field configuration. Future work will assess the impact of using higher ion-electron mass ratio in global two- and three-dimensional PIC simulations and in the bow shock kinetic structure.

\section{ACKNOWLEDGMENTS}

This work was funded by the Swedish VR Grant No. D621-2013-4309 and by the European Commission through the EPiGRAM project (Grant agreement No. 610598. epigramproject.eu). This work used resources provided by the Swedish National Infrastructure for Computing (SNIC) at PDC.

S.M. was partially supported by the Space Hazards Induced near Earth by Large, Dynamic Storms (SHIELDS) project, funded by the U.S. Department of Energy through the Los Alamos National Laboratory Directed Research and Development program.

${ }^{1}$ O. Buneman, T. Neubert, and K.-I. Nishikawa, IEEE Trans. Plasma Sci. 20, 810 (1992).

${ }^{2}$ K.-I. Nishikawa, T. Neubert, and O. Buneman, in Plasma Astrophysics and Cosmology (Springer, 1995), pp. 265-276.

${ }^{3}$ K.-I. Nishikawa, Geophys. Res. Lett. 25, 1609, doi:10.1029/98GL01027 (1998).

${ }^{4}$ K. Nishikawa, Geophys. Monograph Am. Geophys. Union 104, 175 (1998).

${ }^{5}$ D. Cai, X. Yan, K.-I. Nishikawa, and B. Lembège, Geophys. Res. Lett. 33, L12101 (2006).

${ }^{6}$ S. Baraka and L. Ben-Jaffel, J. Geophys. Res.: Space Phys. (1978-2012) 112, A06212 (2007)
${ }^{7}$ D. Cai, W. Tao, X. Yan, B. Lembege, and K.-I. Nishikawa, J. Geophys. Res.: Space Phys. (1978-2012) 114, A12210 (2009).

${ }^{8}$ K.-I. Nishikawa and S. Ohtani, J. Geophys. Res.: Space Phys. (1978-2012) 105, 13017 (2000).

${ }^{9}$ K.-I. Nishikawa and S.-i. Ohtani, IEEE Trans. Plasma Sci. 28, 1991 (2000).

${ }^{10} \mathrm{C}$. C. Goodrich, "Numerical simulations of quasi-perpendicular collisionless shocks," in Collisionless Shocks in the Heliosphere: Reviews of Current Research (American Geophysical Union, 2013), pp. 153-168.

${ }^{11}$ D. Burgess, E. Möbius, and M. Scholer, Space Sci. Rev. 173, 5 (2012).

${ }^{12}$ R. Treumann, Astron. Astrophys. Rev. 17, 409 (2009).

${ }^{13} \mathrm{C}$. Kennel, J. Edmiston, and T. Hada, A Quarter Century of Collisionless Shock Research (Wiley Online Library, 1984).

${ }^{14}$ J. Edmiston and C. Kennel, J. Plasma Phys. 32, 429 (1984).

${ }^{15}$ T. Tanaka, J. Comput. Phys. 111, 381 (1994).

${ }^{16}$ L. K. Daldorff, G. Tóth, T. I. Gombosi, G. Lapenta, J. Amaya, S. Markidis, and J. U. Brackbill, J. Comput. Phys. 268, 236 (2014).

${ }^{17}$ I. B. Peng, S. Markidis, A. Vaivads, J. Vencels, J. Amaya, A. Divin, E. Laure, and G. Lapenta, Proc. Comput. Sci. 51, 1178 (2015).

${ }^{18}$ I. B. Peng, J. Vencels, G. Lapenta, A. Divin, A. Vaivads, E. Laure, and S. Markidis, J. Plasma Phys. 1, 325810202 (2015).

${ }^{19}$ S. Markidis, G. Lapenta, and Rizwan-uddin, Math. Comput. Simul. 80, 1509 (2010).

${ }^{20}$ G. Paschmann, N. Sckopke, I. Papamastorakis, J. Asbridge, S. Bame, and J. Gosling, Journal J. Geophys. Res.: Space Phys. (1978-2012) 86, 4355 (1981).

${ }^{21}$ N. Sckopke, G. Paschmann, S. Bame, J. Gosling, and C. Russell, J. Geophys. Res.: Space Phys. (1978-2012) 88, 6121 (1983).

${ }^{22}$ S. D. Bale, F. S. Mozer, and T. S. Horbury, Phys. Rev. Lett. 91, 265004 (2003).

${ }^{23}$ S. J. Schwartz, E. Henley, J. Mitchell, and V. Krasnoselskikh, Physical Rev. Lett. 107, 215002 (2011).

${ }^{24}$ W. Livesey, C. Kennel, and C. Russell, Geophys. Res. Lett. 9, 1037, doi:10.1029/GL009i009p01037 (1982).

${ }^{25}$ M. Mellott and W. Livesey, J. Geophys. Res.: Space Phys. (1978-2012) 92, 13661 (1987).

${ }^{26}$ C. Russell, M. Hoppe, and W. Livesey, Nature 296, 45-48 (1982).

${ }^{27}$ G. Lapenta and J. King, J. Geophys. Res.: Space Phys. (1978-2012) 112, A12204 (2007).

${ }^{28}$ K.-I. Nishikawa, J. Geophys. Res.: Space Phys. (1978-2012) 102, 17631 (1997).

${ }^{29}$ T. Moore, J. Burch, W. Daughton, S. Fuselier, H. Hasegawa, S. Petrinec, and Z. Pu, J. Atmos. Solar-Terres. Phys. 99, 32 (2013).

${ }^{30}$ C. Wu, D. Winske, Y. Zhou, S. Tsai, P. Rodriguez, M. Tanaka, K. Papadopoulos, K. Akimoto, C. Lin, M. Leroy et al., Space Sci. Rev. 37, 63 (1984).

${ }^{31}$ R. A. Treumann and W. Baumjohann, Advanced Space Plasma Physics (World Scientific, 1997).

${ }^{32}$ M.-B. Kallenrode, Space Physics: An Introduction to Plasmas and Particles in the Heliosphere and Magnetospheres (Springer Science \& Business Media, 2013).

${ }^{33}$ K. Stasiewicz, S. Markidis, B. Eliasson, M. Strumik, and M. Yamauchi, EPL (Europhysics Letters) 102, 49001 (2013).

${ }^{34}$ K. Stasiewicz, Plasma Phys. Controlled Fusion 49, B621 (2007).

${ }^{35}$ H. Hasegawa, M. Fujimoto, T.-D. Phan, H. Reme, A. Balogh, M. Dunlop, C. Hashimoto, and R. TanDokoro, Nature 430, 755 (2004).

${ }^{36}$ W. Baumjohann, R. A. Treumann, and R. A. Treumann, Basic Space Plasma Physics (World Scientific, 1996), Vol. 57. 